\title{
Species composition and distribution of dangerous scorpions of Hemiscorpius genus and clinical symptoms due to envenomation in high-risk regions of southern Iran
}

\author{
MEHRAN SHAHI ${ }^{1}$, SEYED HAMID MOOSAVY ${ }^{2}$, AHMAD ALI HANAFI-BOJD ${ }^{3}$, MORTEZA AKBARI ${ }^{4}$, \\ JAVAD RAFINEJAD ${ }^{3, \vee}$ \\ ${ }^{1}$ Department of Medical Entomology \& Vector Control, School of Public Health \& Infectious and Tropical Diseases Research Center, Hormozgan Health \\ Institute, Hormozgan University of Medical Sciences. Jomhuri Eslami Blvd., Bandar Abbas, Iran \\ ${ }^{2}$ Department of Internal Medicine, Hormozgan University of Medical Science. Jomhuri Eslami Blvd., Bandar Abbas, Iran \\ ${ }^{3}$ Department of Medical Entomology \& Vector Control, School of Public Health, Tehran University of Medical Sciences. Tehran, Iran \\ ${ }^{4}$ Department of public health, School of Health, Ilam University of Medical Sciences. Ilam, Iran
}

Manuscript received: 27 May 2021. Revision accepted: 26 June 2021

\begin{abstract}
Shahi M, Moosavy SH, Hanafi-Bojd AA, Akbari M, Rafinejad J. 2021. Species composition and distribution of dangerous scorpions of Hemiscorpius genus and clinical symptoms due to envenomation in high-risk regions of southern Iran. Biodiversitas 22: 2945-2951. The southern regions of Iran, including Hormozgan province, are high risk areas for scorpion stings. This study aimed to determine the distribution and species diversity of dangerous scorpions of Hemiscorpius and also to determine the clinical symptoms of their bites in the high-risk areas of Hormozgan province. Scorpion sampling was carried out from Shahid Mohammadi and Koodakan hospitals in Bandar Abbas also filed areas of Bandar Abbas and Khamir counties by searching the scorpion's shelter throughout the day and using ultraviolet light at night. To determine the clinical symptoms, the data recorded in the patient's file were extracted and recorded in the sting checklists. Five species of the Hemiscorpius genus were identified. H. gaillardia and H. persicus are reported for the first time from Hormozgan province. Specific clinical symptoms after several hours of delayed were pain, swelling, itching, warmth, and edema, necrosis of the sting site, nausea, vomiting, dizziness, and fever. Complications such as urine discoloration, hemoglobinuria, severe hemolysis, and acute renal failure were observed. The results showed that envenomation with Hemiscorpius Scorpions should be considered as an urgent medical issue.
\end{abstract}

Keywords: Antivenom, hemoglobinuria, Iran, renal failure, scorpion sting

\section{INTRODUCTION}

Scorpions are among the poisonous arthropods and every year in warm seasons in large areas especially western and southern regions of Iran threatens the health of many residents of these areas. Annually about 50,000 cases of scorpion sting are reported in Iran (Mousavi et al. 2015). Most of the deaths due to scorpion stings occur in the western and southern regions of Iran (Dehghani Rouhullah and Fathi 2012, Karami et al. 2013, Shahi Mehran et al. 2015). H. lepturus is the most dangerous scorpion species in western and southwestern regions of Iran (Dehghani R et al. 2012, ZARE et al. 2010). This scorpion has cytotoxic venom and causes complications such as hemolysis, renal failure, and sometimes death in patients (Dehghani R et al. 2012). Khuzestan, Hormozgan, Sistan and Baluchestan, and Kerman provinces are the most important scorpion envenomation centers in Iran (Dehghani Rouhullah et al. 2016). Hormozgan province is one of the high-risk scorpion-sting in southern Iran (Rafinejad et al. 2020). Every year significant sting cases of scorpion and death due to scorpion envenomation are reported in this province (Moosavy et al. 2016, Shahi Mehran et al. 2015). In 2018, 4292 scorpion stings and 5 cases of deaths induced by scorpion envenomations were reported in the Hormozgan province that Bandar Abbas with 1307 cases were the most related (Shahi Mehran et al. 2021). Unfortunately, considering the importance of scorpion envenomation, very few studies have been conducted on fauna, ecology, epidemiology, and clinical symptoms of scorpions in this province.

So far 22 scorpion species have been reported from different regions of Hormozgan province that 5 species belonging to the Hemiscorpius genus including $H$. persicus, $H$. lepturus, $H$. enischnochela, $H$. acanthocarcus, and $H$. gaillardia that they are among the most dangerous scorpions in Iran (Navidpour et al. 2013, Rahmani et al. 2015, Shahi Mehran et al. 2016). Hemiscorpius sting may cause a wide variety of clinical symptoms in the patients which include fever, allergies, salivation, vomiting, seizure, nervous system failure, hemolysis, acute renal failure, cardiovascular failure, edema, blister, and necrosis that in some cases cause death (Shahi Mehran et al. 2020a).

Considering the importance of scorpion envenomation and lack of information from the main agent of sting and death of patients in some parts of the province, this descriptive study was designed and conducted. The main purpose of this study was to identify dangerous species of scorpions and their distribution in high-risk cities in terms of scorpion sting including Bandar Abbas and Khamir and 
determine the clinical symptoms of envenomation to urgent referral, timely care, and treatment, and prevent severe complications.

\section{MATERIALS AND METHODS}

\section{Study area}

This is a cross-sectional study that conducted in Bandar Abbas and Khamir counties, which are considered among the most high-risk centers in terms of scorpion stings and death due to scorpion envenomation in Hormozgan province and also was performed on scorpion sting victims referred to emergency wards of Shahid Mohammadi and Koodakan hospitals of Bandar Abbas during 2014-2015.

Scorpion sampling was done by determining sampling stations in different parts of the mentioned Counties (Figure 1). To determine the causes of the sting and clinical symptoms in the patients, the samples of scorpions caught by the patients were collected at the time of admission, and clinical information was recorded from the patients' file and registering in the regulated checklists.

Bandar Abbas County has located in geographical coordinates of $54^{\circ}$ and 53 minutes to $56^{\circ}$ and 30 minutes east length and $26^{\circ}$ and $53 \mathrm{~min}$ to $27^{\circ}$ and $31 \mathrm{~min}$ north width on the southern coast of Iran. Bandar Abbas is situated on flat ground with an average altitude of 9 meters above sea level. The mean rainfall in Bandar Abbas County was reported between 150 and $200 \mathrm{~mm}$ per year (16). Khamir County with an area of $3705.9 \mathrm{~km}^{2}$ consists of two parts about $5.2 \%$ of the total soil area of Hormozgan province. The county is located in geographical coordinates of $53^{\circ}$ and 49 minutes to $55^{\circ}$ and 23 min east length and $26^{\circ}$ and $54^{\circ}$ to $27^{\circ}$ and 28 minutes north width. The average annual rainfall is about $120 \mathrm{~mm}$, and the annual relative humidity is about $75 \%$.

\section{Sampling size and scorpion identification}

Sampling was done by random sampling method during the day (Until noon) from under the rocks and dried tree branches and night time (Night catch with UV light) from 20 villages of Bandar Abbas and Khamir counties during 2014-2015. The collected scorpions were placed in a container containing $75 \%$ alcohol with identification labels. Samples were identified by Nikon stereomicroscope XNModel using diagnostic keys of Iranian scorpions in the medical entomology laboratory of Bandar Abbas School of Public Health (Monod and Lourenco 2005, Navidpour et al. 2013). Data were analyzed by SPSS 16 software using descriptive and inferential statistics including frequency, percentage, and k-squire. Tables and charts were plotted by Excel software. ArcGIS 10.2 software was used to determine the spatial distribution of dangerous Hemiscorpius species in the studied areas.

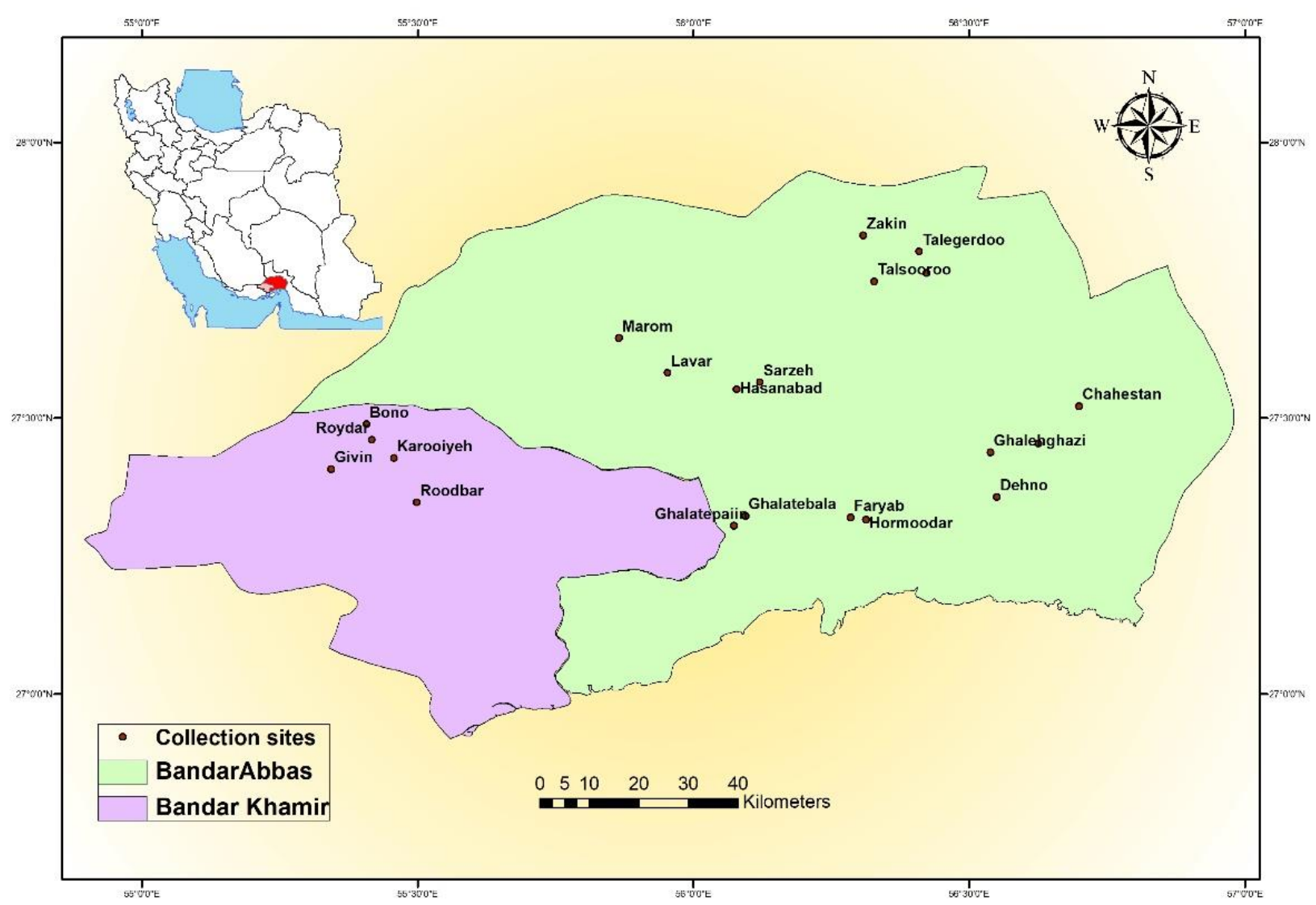

Figure 1. Sampling stations in the studied areas in Bandar Abbas and Khamir Counties, Iran, 2014 


\section{RESULTS AND DISCUSSION}

In total, 18 scorpion species were identified from 551 samples collected from the studied areas. (Table 1). The highest species richness was identified in Hemiscorpius scorpions with the five species in Bandar Abbas. In Khamir and Bandar Abbas Counties, the dominant species in Hemiscorpius genus were $H$. acanthocercus and $H$. enischnochela respectively. In this study, for the first time, $H$. persicus and $H$. gaillardia species were identified and reported from Hormozgan province. Mesobuthus and Odontobuthus genus had the highest abundance. Orthochirus, and Razianus genus had the lowest abundance in the studied areas (Figure 2).

\section{Scorpion Species Identification}

Laboratory studies on 305 samples of scorpions, brought by afflicted patients showed that these scorpions belonged to eight genera and 16 species. Hemiscorpius genus with five species was the most frequent and the genus Rzianos had the lowest frequency with one species. H.acantosercos was the dominant species. (Table 2).

The number of antivenoms used in the patients is determined by scorpion species. The highest Scorpion antivenom was used in the Hemiscorpius and Compsobuthus envenomation (Table 2).

Table 2. Genus and species composition and Frequency of antivenom used in the treatment of envenomation scorpions in Shahid Mohammadi and Koodakan Hospitals of Bandar Abbas, Iran, 2014

\begin{tabular}{llcccc}
\hline \multirow{2}{*}{ Family } & \multicolumn{1}{c}{$\begin{array}{c}\text { Genus or } \\
\text { species }\end{array}$} & \multicolumn{2}{c}{$\begin{array}{c}\text { Scorpion } \\
\text { No. }\end{array}$} & \multicolumn{2}{c}{ Antivenom } \\
No. & $\%$ \\
\hline Hemiscorpiidae & Hemiscorpius & 67 & 22 & 153 & 21 \\
& acanthocercus & & & & \\
& H. enischnochela & 49 & 16.1 & 125 & 17 \\
& H. lepturus & 5 & 1.6 & 12 & 1.6 \\
& H. persicus & 4 & 1.3 & 10 & 1.4 \\
& H. gaillardia & 3 & 1 & 9 & 1.2 \\
Buthidae & & & & \\
& Compsobuthus & 57 & 18.7 & 136 & 18.6 \\
& Mesobuthus & 37 & 12.1 & 87 & 12 \\
& Androctonus & 28 & 9.2 & 72 & 10 \\
& Odontobuthus & 19 & 6.2 & 42 & 5.7 \\
& Orthochirus & 19 & 6.2 & 38 & 5.2 \\
Total & Hottentotta & 16 & 5.2 & 44 & 6 \\
& Razianus & 1 & 0.3 & 2 & 0.3 \\
& & 305 & 100 & 730 & 100 \\
\hline
\end{tabular}

Table 1: Distribution and scorpion species composition in Bandar Abbas and Khamir counties, Iran, 2014

\begin{tabular}{|c|c|c|c|c|c|c|c|c|c|c|c|c|c|c|c|c|c|c|c|}
\hline Areas understudy & Village & 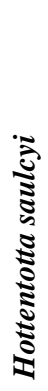 & 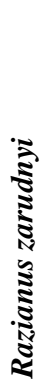 & 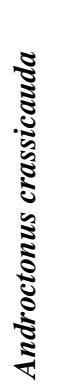 & 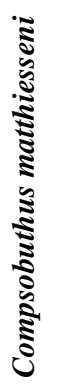 & 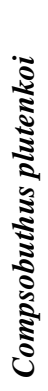 & 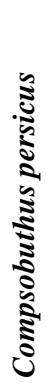 & 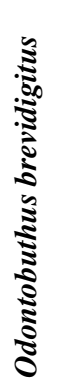 & 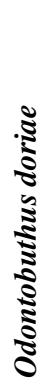 & 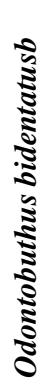 & 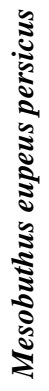 & 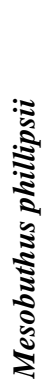 & 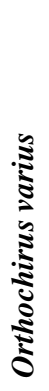 & 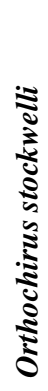 & 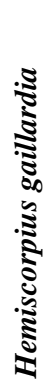 & 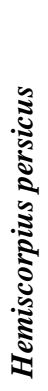 & 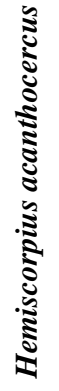 & 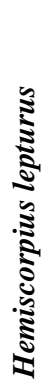 & 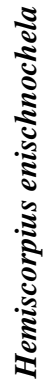 \\
\hline \multirow[t]{8}{*}{ Fin } & Zakin & + & - & - & - & + & + & - & - & - & + & - & - & + & - & - & + & - & + \\
\hline & Talegerdoo & + & - & + & - & - & + & - & + & - & + & + & - & + & - & + & + & + & + \\
\hline & Lavar & - & - & + & + & + & + & - & + & - & + & + & - & - & - & - & + & - & + \\
\hline & Sarzeh & - & - & + & - & - & - & - & + & - & + & - & - & - & - & - & - & - & - \\
\hline & Islamabad & - & - & - & - & - & + & - & + & + & + & - & - & - & - & - & + & - & - \\
\hline & Talsuroo & - & - & + & - & - & + & - & + & - & + & + & - & + & - & - & - & - & - \\
\hline & Hassanabad & - & - & - & - & - & + & - & + & - & + & - & - & + & - & - & + & - & + \\
\hline & Marom & - & - & - & - & - & - & - & + & + & + & + & - & - & - & - & + & - & - \\
\hline Markazi Takht \& & Ghalatebala & - & + & - & - & - & + & - & + & - & + & + & - & + & - & - & + & + & + \\
\hline \multirow[t]{7}{*}{ Ghaleh gfhazi } & Faryab & - & - & + & - & - & + & - & - & - & - & - & - & - & - & - & + & - & - \\
\hline & Chahestan & - & & + & - & - & + & - & + & - & + & - & - & + & + & + & + & - & + \\
\hline & Ghalehghazi & - & - & + & - & - & + & + & + & - & + & + & - & - & - & - & + & - & - \\
\hline & Ghalatepain & - & - & - & - & - & + & - & + & - & + & - & - & - & + & - & + & - & - \\
\hline & Hormoodar & - & - & + & - & - & - & - & + & - & + & + & - & + & - & - & + & - & - \\
\hline & Sayedsoliman & - & - & - & + & - & - & - & + & - & + & + & - & - & - & - & - & - & - \\
\hline & Dehno & - & - & + & - & - & - & - & + & + & + & - & - & - & - & - & - & - & - \\
\hline \multirow[t]{4}{*}{ Roodar } & Bono & + & - & - & - & + & + & - & + & - & + & + & - & + & - & - & + & + & + \\
\hline & Keruieyeh & + & - & - & - & - & + & - & - & - & + & + & + & - & - & - & + & - & + \\
\hline & Gevin & - & - & - & - & - & + & & + & - & + & + & - & - & - & - & + & - & + \\
\hline & Roodbar & - & - & + & - & - & - & - & + & - & + & - & - & - & - & - & - & - & + \\
\hline
\end{tabular}



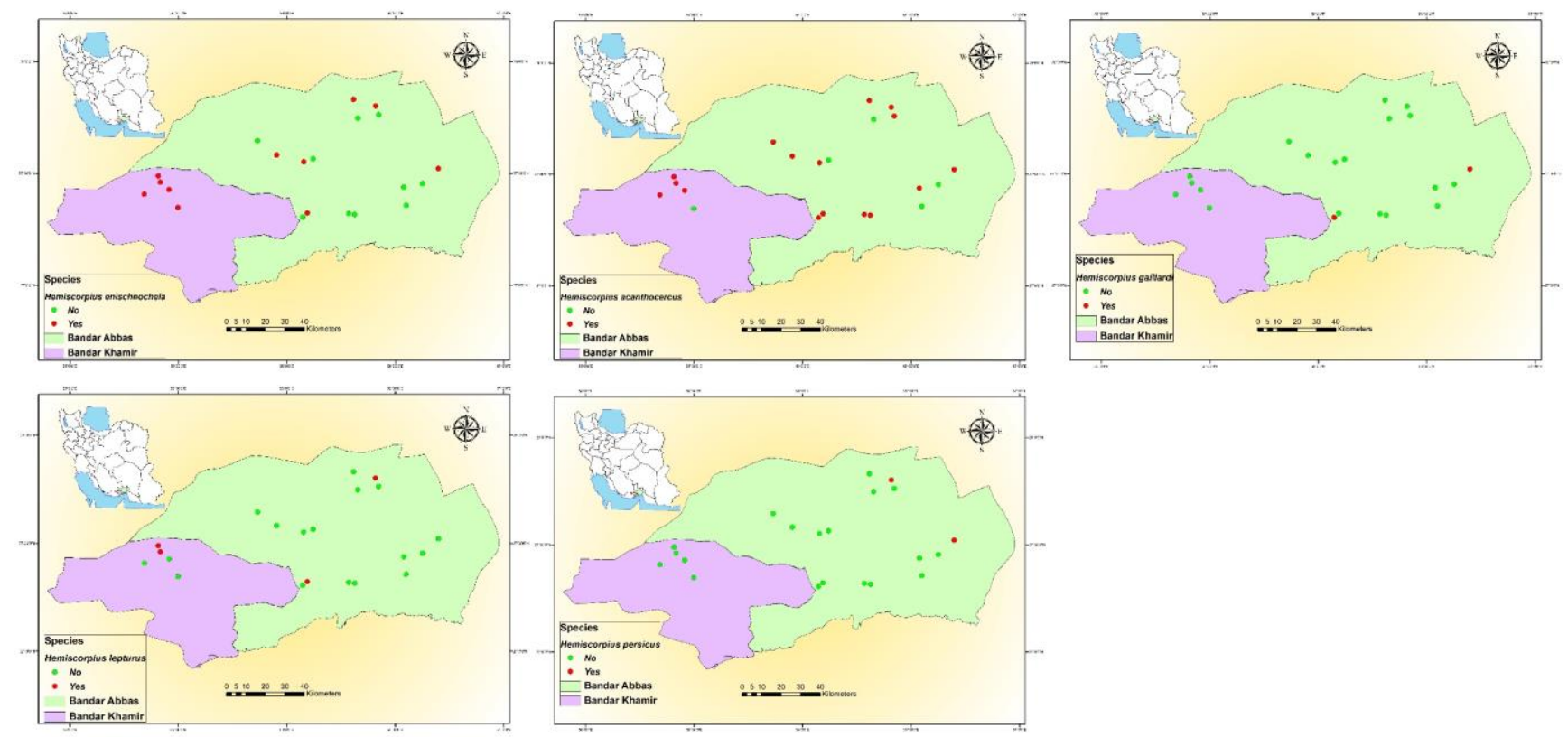

Figure 2. Species distribution of Hemiscorpius in Bandar Abbas and Khamir counties, Iran, 2014

\section{Clinical symptom}

In this study, a total of 305 patients including 149 men and 156 women were examined. Clinical findings of patients are shown in Figure 3. The most common clinical symptoms of scorpion sting in patients were redness and the least symptoms were related to Rash (Figure 3).

The recorded reports and observations indicated a lack of complaints from pain and burning in the site of sting in the early stages of Hemiscorpius scorpion sting. After a few hours, redness, pain, and other clinical symptoms are common.

Other symptoms with lower percentage selected by Hemiscorpius scorpions were Sweating (2\%), muscle pain (1.6\%), hand and foot tingling (1.6\%), cellulite $(1.3 \%)$, Numbness of bite organ (1\%), tachycardia $(0.7 \%)$, shortness of breath $(0.7 \%)$, Chest pain $(0.7 \%)$, Blurred vision $(0.7 \%)$, epigastric burning $(0.7 \%)$, and generalized rash $(0.3 \%)$, Confusion $(0.3 \%)$ and Weakness $(0.3 \%)$. Among the victims, three (1\%) were studied. Among the patients, three $(1 \%)$ were allergic to scorpion antidote serum. Also, five $(1.6 \%)$ of the patients were admitted to the intensive care unit (ICU) due to acute symptoms such as hematuria and severe hemolysis.

\section{Discussion}

The highest species richness of scorpions was related to mountainous areas. This finding is consistent with the results of other similar studies (Azizi et al. 2001). In Bandar Abbas, the highest species richness in Hemiscorpius genus was observed by the identification of five species of $H$. acanthocercus, $H$. enischnochela, $H$. gaillardia, H. persicus, and $H$. lepturus. Also, the highest frequency was related to $H$. acanthocercus. This species is native to Hormozgan province (Monod and Lourenco 2005).

In Khamir County, only three species of Hemiscorpius were identified including $H$. enischnochela, $H$. acanthocercus, and $H$. lepturus. The dominant species of Hemiscorpius was $H$. enischnochela in this region. The high frequency of patients envenomed by $H$. acanthocercus and $H$. enischnochela referred to the Shahid Mohammadi, and Koodakan hospitals, confirms the accuracy of the results of the field (Shahi Mehran et al. 2020b).

Hemiscorpius enischnochela was abundant in rural areas of Khamir County. This is one of the species that tend to enter human residences, especially in summer (Shahi M et al. 2009). This can be due to the escape from the heat and sheltering into a cool and wet environment of human places. Endophilly behavior of this species increases their risk of contact with residents of these places.

The highest geographical distribution in the studied areas is related to $H$. acanthocercus. This scorpion was collected in major locations of collection. The dominant species of scorpions belonging to genus Hemiscorpius in mountainous areas in Bandar Abbas and Khamir counties were $H$. acanthocercus and $H$. enischnochela respectively.

Hemiscorpius lepturus were collected from two stations in Bandar Abbas and Khamir counties. H. gaillardia and $H$. persicus were observed only in central, fin, and Takht parts of Bandar Abbas County with very low frequency. In Hormozgan province, except for two species of $H$. gaillardia and $H$. persicus, which are first reported from Hormozgan province, the other species of Hemiscorpius including $H$. acanthocercus, $H$. enischnochela, and $H$. lepturus has been reported by different researchers (Monod and Lourenco 2005; Navidpour et al. 2012; Navidpour et al. 2013). 


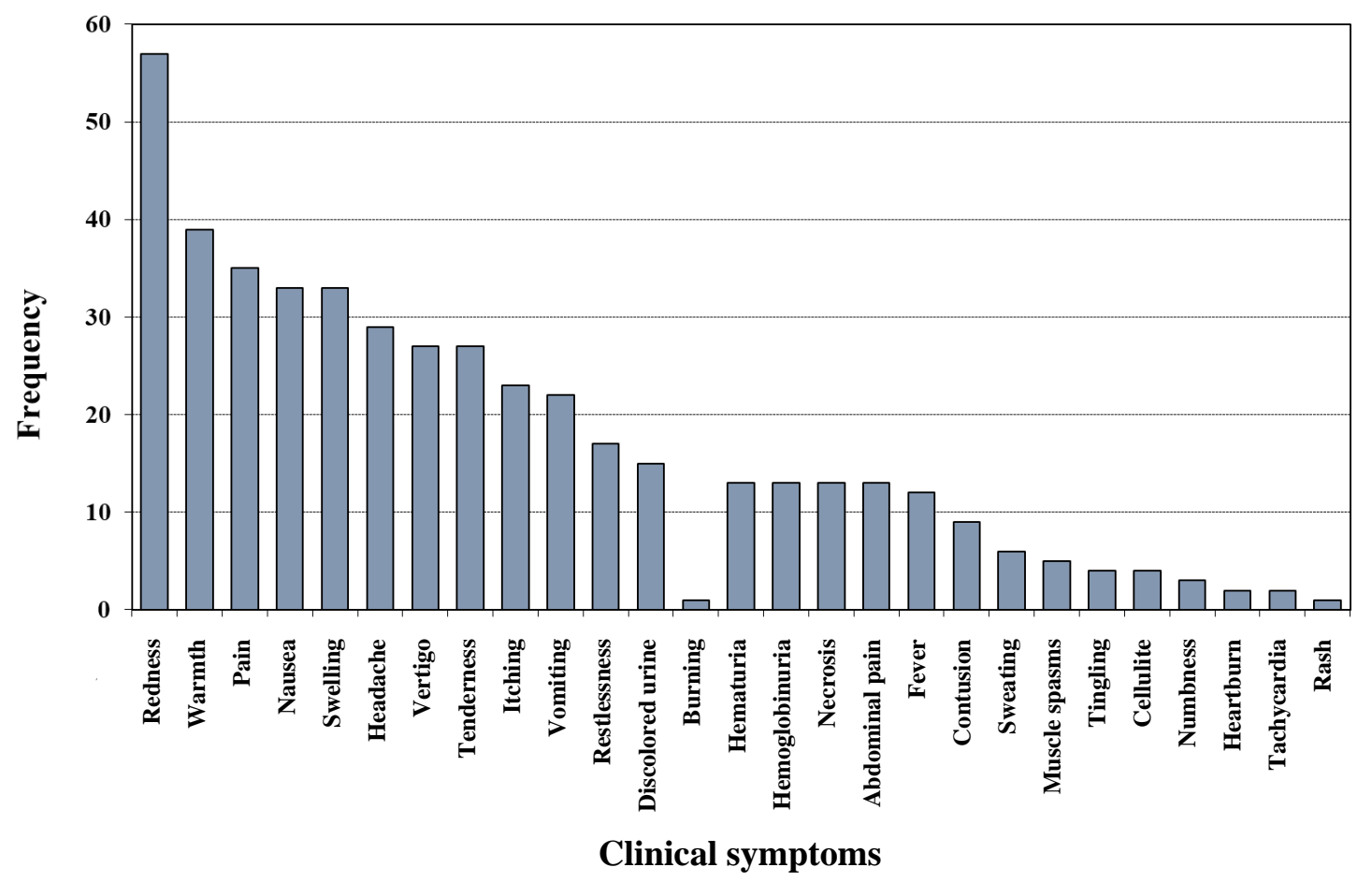

Figure 3. Frequency of specific clinical symptoms observed in patients selected by Hemiscorpius scorpions, Shahid Mohammadi, and Koodakan hospitals of Bandar Abbas, Iran, 2014

Compsobuthus persicus is another scorpion identified with high abundance and envenomation cases in the patients. This scorpion was the second cause of scorpion sting in the patients under investigation.

Other species with high frequency were Mesobuthus eupeus, Odontobuthus doriae, and respectively. In another study in Jask County of Hormozgan province, the dominant species was Mesobuthus eupeus (Fekri et al. 2012). The rarest species in this study were Odontobuthus brevidigitus, Razianus zarudnyi, and Orthochirus varius. Two recent species have been reported by Navidpour from Hormozgan province (Navidpour et al. 2013).

In this study, dangerous species of Hemiscorpius include $H$. acanthocercus, $H$. enischnochela, $H$. persicus, and $H$. gaillardia are first introduced as scorpion sting agents in the south of Iran. Hemiscorpius persicus and $H$. gaillardia have been reported only from the east of Iran (Monod and Lourenco 2005). Hemiscorpius lepturus is one of the most dangerous scorpions identified in Khuzestan province (Mohseni et al. 2013, Rahmani and Jalali 2012). In this study, $H$. acanthocercus had the highest rate of scorpion sting in the patient's people. Hemiscorpius gaillardia scorpion had the least frequency of scorpion sting in Hemiscorpius genus.

A wide range of clinical symptoms in patients was observed including, burning, redness or stinging site, nausea, dizziness, and headache. Other clinical symptoms with less frequency were swelling, itching, vomiting, fever, abdominal pain, restlessness, necrosis, and hematuria. In similar studies in western parts of Iran, these clinical symptoms have been reported in scorpion envenomation patients (Rahmani and Jalali 2012).

Out of 305 patients, five $(1.6 \%)$ were admitted to the intensive care unit (ICU) due to acute symptoms including hematuria and severe hemolysis. All of these victims were stung by species belonging to Hemiscorpius including $H$. enischnochela and $H$. acanthocercus. So far in western regions of Iran, only one species of scorpion belonging to the genus Hemiscorpius has been introduced as the cause of necrosis, hemolysis and severe hematuria, and even death (Vazirianzadeh et al. 2013).

No acute symptoms such as hematuria, hemolysis, and renal failure were observed in the patients who were stung by Buthidae family. The clinical symptoms of scorpion sting are very different and according to scorpion species, the amount of venom injected, season, age and physiological status of the injured may occur from a mild local reaction to severe physiological changes leading to death (Rafizadeh et al. 2013).

According to the statements of the patients, unlike the Buthidae family, stinging of Hemiscorpius scorpions was associated with very little pain in the sting site. Some researchers also reported the mild pain of the sting with Hemiscorpius (Dehghani R et al. 2012, Vazirianzadeh et al. 2013). Hemiscorpionidae family has a small sting of about $1 \mathrm{~mm}$ and their sting have a low pain. This causes a lack of attention and causes delayed referral to hospital and treatment.

Our finding showed, in envenomation with Hemiscorpius, the pain was created after at least four hours 
which was associated with redness, swelling, and itching of the stinging site. Other symptoms such as nausea, vomiting, dizziness, headache, inflammation, transpiration, and abdominal pain were observed exclusively or with much more frequency than the patients who were stung by other genus scorpions. In addition, in envenomation with Hemiscorpius scorpions, necrosis of the sting site, urine discoloration, and hemoglobinuria were observed. The important point is that in the patients with plurality sting or when the place of the sting is in the trunk area, the symptoms had been found faster. The results of another study showed that renal failure was more severe in children who were stung in the trunk and delayed referring to the hospital (Afzali and Pezeshki 1998).

According to reports in scorpion patients in western Iran, the most severe complications observed due to $H$. lepturus (Kassiri et al. 2012a, Vazirianzadeh et al. 2013). The results of different researchers' studies have shown that $H$. lepturus venom has neurotoxic, cytotoxic, and hemolytic effects and causes severe skin wounds and inflammations with hidden and severe hemolysis (Dehghani R et al. 2012, ZARE et al. 2010). It also affects the central nervous system, the cardiovascular, skin, and kidneys (Emam et al. 2011). In the present study, severe complications such as severe hemolysis, hematuria, necrosis, acute renal failure, and death with mentioned acute symptoms were observed.

The main strategy for scorpion sting treatment in Iran is using specific antidotes of any species (Kassiri et al. 2012a, Kassiri et al. 2012b). The polyvalent serum has been prepared specifically for the treatment of envenomation of six dangerous scorpion species in Iran. The important point is that currently the polyvalent serum in Iran containing only the antidote of one of the Hemiscorpius species $(H$. lepturus). Due to the necessity of specificity of the antidote for each species, according to the available evidence, this antidote is not specific for the treatment of patients people bitten by other species of the Hemiscorpius and does not have the effectiveness.

Our finding in this study showed that Hemiscorpius sting should be considered as a medical emergency. This requires immediate attention based on clinical symptoms in the injured. It is also recommended that physicians in this region become familiar with the scorpions of the region and their treatment protocol.

Due to the importance of the subject, it is very important and necessary to conduct more extensive clinical studies and also to investigate the distribution of dangerous species belonging to the Hemiscorpius genus in other parts of the province.

In conclusion, the results showed that envenomation with Hemiscorpius scorpions should be considered as an urgent medical issue. In view of the importance of the subject, it is suggested that studies on Hemiscorpius venom and the evaluation of the effect of scorpion anti-venom should be taken.

\section{ACKNOWLEDGEMENTS}

Hereby thank the colleagues of the emergency department and medical records of Shahid Mohammadi and Koodakan Hospitals of Bandar Abbas who helped us in conducting this study and also from the research deputy of Hormozgan University of Medical Sciences for financial support in conducting this research. This study was funded by Vice-Chancellor for Research and Technology, Hormozghan University of Medical Sciences, Iran.

\section{REFERENCES}

Afzali N, Pezeshki N. 1998. Surveying of kidney acute dysfunction following Hemiscorpius lepturus sting in children. J Med Sci 25: 4248.

Azizi K, Shahraki G, Omrani M. 2001. Determination of the fauna and sex ratio of scorpions from villages and suburbs of Kohgilouieh and Boirahmad province in 1379. J Armaghan Danesh 6: 21-22.

Dehghani R, Fathi B. 2012. Scorpion sting in Iran: a review. Toxicon 60: 919-933.

Dehghani R, Haghi FM, Mogaddam MY, Sedaghat M, Hajati H. 2016. Review study of scorpion classification in Iran. J Entomol Zool Stud 4: 440-444.

Dehghani R, Khamechian T, Vazirianzadeh B, Vatandoost H, Moravvej S. 2012. Toxic effects of scorpion, Hemiscorpius lepturus (Hemiscorpiidae) venom on mice. J Anim Plant Sci 22: 593-596. DOI: 10.1016/j.toxicon. 2012.06.002

Emam SJ, Malihi R, Jafari Mosavi SAA, Vazirianzadeh B, Visi I. 2011. Evaluation of hematological and urine parameters in Hemiscorpius lepturus (Gadim) victims referred to Health Centre of Hendijan, sw Iran. Jundishapur J Health Sciences 3:-.

Fekri S, Badzohre A, Safari R, Azizi K. 2012. Species identification and geographic distribution of scorpions in Jask town county, Hormozgan province. Hormozgan Med J 16: 135-142.

Karami K, Vazirianzadeh B, Mashhadi E, Hossienzadeh M, Abbas Moravvej S. 2013. A five year epidemiologic study on scorpion stings in Ramhormoz, South-West of Iran. Pakistan J Zool 45 (2): 469-474.

Kassiri H, Mohammadzadeh Mahijan N, Hasanvand Z, Shemshad M, Shemshad K. 2012a. Epidemiological survey on scorpion sting envenomation in South-West, Iran. Zahedan J Res Med Sci 14: 80-83.

Kassiri H, Teimouri A, Shemshad M, Sharifinia N, Shemshad K. 2012 b. Epidemiological survey and clinical presentation on scorpionism in south-west of Iran. Middle East J Sci Res 12: 325-330. DOI: 10.5829/idosi.mejsr.2012.12.3.64113

Mohseni A, Vazirianzadeh B, Hossienzadeh M, Salehcheh M, Moradi A, Moravvej SA, Goodacre S. 2013. The roles of some scorpions, Hemiscorpius lepturus and Androctonus crassicauda, in a scorpionism focus in Ramhormorz, southwestern Iran. J Insect Sci 13 (1): 89. DOI: 10.1673/031.013.8901.

Monod L, Lourenco WR. 2005. Hemiscorpiidae (Scorpiones) from Iran, with descriptions of two new species and notes on biogeography and phylogenetic relationships. Revue Suisse de Zoologie 112: 869-942. DOI: $10.5962 /$ bhl.part.80331.

Moosavy SH, Shahi M, Rafinejad J, Zare S, Madani A, Navidpour S. 2016. Epidemiological aspect of scorpion sting in Bandar Abbas, Iran, during 2009-2011. Electr Physician 8:2286. DOI: 10.19082/2286.

Mousavi H, Navidpour S, Rafinejad J. 2015. A review study on distribution and medical importance of Hemiscorpius Peters, 1861 in Iran. J Mazandaran University of Medical Sciences 24:107-124.

Navidpour S, Fet V, Kovařík F, Soleglad ME. 2012. Scorpions of Iran (Arachnida, Scorpiones). Part VIII. Fars Province. Euscorpius 2012:1-29. DOI: 10.18590/euscorpius.2012.

Navidpour S, Soleglad ME, Fet V, Kovařík F. 2013. Scorpions of Iran (Arachnida, Scorpiones). Part IX. Hormozgan province, with a description of Odontobuthus tavighiae sp. n.(Buthidae). Euscorpius 2013: 1-29. DOI: 10.18590/euscorpius.2013

Rafinejad J, Shahi M, Navidpour S, Jahanifard E, Hanafi-Bojd AA. 2020. Effect of climate change on spatial distribution of scorpions of significant public health importance in Iran. Asian Pac J Trop Med 13: 503. DOI: $10.4103 / 1995-7645.295361$ 
Rafizadeh S, Rafinejad J, Rassi Y. 2013. Epidemiology of scorpionism in Iran during 2009. J Arthropod Borne Dis 7 (1): 66-70.

Rahmani A, Forouzandeh H, Kalantar M, Asad-Masjedi N, Alavian Z, Kavarizadeh K. 2015. Epidemiological and clinical characteristics of scorpion stings in Ahwaz, Southwest Iran (2006-2010). Intl J Med Toxicol Forens Med 5: 201-206. DOI: 10.22037/ijmtfm.v5i4(Autumn).9442.

Rahmani A, Jalali A. 2012. Symptom patterns in adult patients stung by scorpions with emphasis on coagulopathy and hemoglubinuria. J venomous animals and toxins including tropical diseases 18: 427-431 DOI:10.1590/S1678-91992012000400011.

Shahi M, Azizi K, Ansarian N. 2009. Study on scorpions fauna in high risk area of Hormozgan province, 2006-7. Hormozgan Med J 12: 207 214

Shahi M, Davoodian P, Davaridolatabadi N, Shahi M. 2020a. A cause of hematuria following Hemiscorpius acanthocercus (Scorpiones: Hemiscorpiidae) Sting in South of Iran. Hormozgan Med J 24. DOI: 10.5812/hmj.95481

Shahi M, Jaberhashemi SA, Hanafi-Bojd AA, Akbari M, Rafinejad J. 2021. Faunistic study on scorpions and their health impact in
Bashagard County, Hormozgan Province, Southern Iran. Nusantara Biosci 13. DOI: $10.13057 /$ nusbiosci/n130112.

Shahi M, Moosavy SH, Hanafi-Bojd AA, Navidpour S, Zare S, Madani A, Rafinejad J. 2016. Spatial distribution of scorpion sting in a high-risk area of southern Iran. J Med Entomol 53: 1198-1204. DOI: 10.1093/jme/tjw043.

Shahi M, Moosavy SH, Sanaei-Zadeh H. 2020b. Severe hemoglobinuria due to Hemiscorpius enischnochela (Scorpiones: Hemiscorpiidae) envenomation from South of Iran. J Emergency Practice Trauma 6: 121-125. DOI: 10.34172/JEPT.2020.22.

Shahi M, Rafinejad J, Az-Khosravi L, Moosavy SH. 2015. First report of death due to Hemiscorpius acanthocercus envenomation in Iran: Case report. Electr Physician 7:1234. DOI: 10.14661/1234.

Vazirianzadeh B, Hossienzadeh M, Moravvej S, Vazirianzadeh M, Mosavi S. 2013. An epidemiological study on scorpion stings in Lordegan County, south-west of Iran. Arch Razi Inst 68 (1): 71-76. DOI: 10.7508/ari.2013.01.012.

ZARE MA, Mahmoudi KS, Teymourzadeh S, Sabiri GH. 2010. Serum enzymes studies in scorpion (Hemiscorpius lepturus) dose-related envenomation in rabbits. Arch Razi Inst 65 (2): 83-89. DOI: 10.22092/ARI.2010.103854. 